\title{
Tendon transfers in tetraplegic patients: The Cleveland experience
}

\author{
Alvin A Freehafer \\ 1746 Dogwood Drive, Marco Island, Florida 34145, USA
}

\begin{abstract}
The author has taken care of patients with spinal cord injury for over 30 years. A major part of these years was spent doing tendon transfers on the patients with tetraplegia. Thirteen were not better, but not worse out of the 285 patients who had an operation. All of the patients have had a period of rehabilitation, waited 1 year following spinal injury, and had a stable neurological examination. The goals of the surgery were wrist extension, elbow extension, thumb pinch, and finger grasp. This was based on the author's classification of the characteristic muscle strengths that are present in the completely paralysed tetraplegic patient. One group of patients had weak or no wrist extension. These patients needed a transfer of the brachioradialis to the extensor carpi radialis brevis. Some of these patients required a posterior deltoid transfer to act as an obstacle to the brachioradialis. The next group of patients had no elbow extension, but had brachioradialis and two radial wrist extensors. They required a posterior deltoid transfer, and an opponens transfer and a transfer to give finger flexion. The next group had elbow extension, brachioradialis, two radial wrist extensors and a pronator teres, and needed an opponens transfer, and a transfer to give finger flexion. The last group of patients had poor or no opposition, and required an opponens transfer.
\end{abstract}

Keywords: spinal cord injury; tendon transfers; wrist extension; elbow extension, opponens transfer; finger flexion transfer

\section{Introduction}

The author has taken care of patients with spinal cord injury for more than thirty years. During this time many tetraplegic patients have had operations carried out on their upper limbs by the author. Thirteen are no better, but the others have been improved. No-one is worse. There have been four evaluations done since 1974. ${ }^{1-4}$ The assessments have all been different and they have all been good. All of the patients have had a period of rehabilitation, waited one year from spinal injury, and have had a stable neurological examination.

The goals of the surgery were active wrist extension, elbow extension, thumb pinch, and finger grasp, and were based on the author's classification of the characteristic muscle strengths that are present in those with complete tetraplegia. Moberg's Classification $^{5}$ or the International Classification fails to get to the point and includes many aspects that are not relevant and should be discontinued in the author's opinion. The author's opinion is that no patient without any sensation present should have a tendon transfer.

Correspondence: A Freehafer

\section{Methods}

The author's classification is as follows:

Group Surgery

I Deltoid transfer occasionally

II Deltoid Transfer

Brachioradialis Transfer for wrist extension

III Deltoid Transfer

Transfer for opposition and finger flexion

IV Transfer for opposition and finger flexion

V Transfer for opposition

The patients in Groups II to V represent those who have complete tetraplegia. These patients are those who can be operated upon and those who can be expected to obtain a good result. They represent neurological levels of C5, C6, C7 and C8.

Most C5 patients or those in Group I have no active function in their hands. A small number with C5 paralysis will have the brachioradialis, extensor carpi radialis longus, and the extensor carpi radialis brevis, but too weak to be useful. These patients may benefit from transfer of the brachioradialis and the extensor carpi radialis longus to the extensor carpi radialis brevis to provide wrist extension. The wrist extensors have different moment arms so the extensor that is in the middle is the one to get the transferred muscles. The author would recommend a posterior deltoid transfer to stabilize the upper limb and to give 
counter balance to the muscle below the elbow. This will give improved and consistent wrist extension. ${ }^{6}$

Patients with $\mathrm{C} 6,7$, and 8 tetraplegia will benefit from the transfers listed in the classification.

\section{Results}

Opponens transfers were done 180 times. Transfers for finger flexion were done 161 times. Posterior deltoid transfers were done 59 times. Transfers for wrist extension were done 17 times.

Moberg has brought to our attention the posterior deltoid transfer. ${ }^{5}$ It was first mentioned by Merle d'Aubigne, and was mentioned by Joseph Boyes in Bunnell's book on hand surgery. ${ }^{7,8}$

Figures 1a, b, c and d show the results following the posterior deltoid transfer. This is a very simple operation which usually lasts for an hour. This procedure has been discussed in detail previously in the Journal of Hand Surgery, American Volume.

Wrist extension procedures helped in seven cases, but no patient had a posterior deltoid transfer. The author recommends this operation for wrist extension in Group II patients. Opponens transfers worked very well and are highly recommended. All of the opponens transfers were done the same way in all but three cases.

Transfers for finger flexion were done the same way and the patients liked this procedure. Figure $2 \mathrm{a}$ and $\mathrm{b}$ show the result in a patient in group III.

Figure $3 \mathrm{a}-\mathrm{d}$ show how the tendons are sutured for opponens and finger flexion transfers. Figure $4 a$ and $b$ show a group IV patient with opponens and finger flexion transfers. Figure $5 \mathrm{a}$ and $\mathrm{b}$ show the result of $\mathrm{a}$ patient in group V. Most of the details about these procedures are described in the Journal of Hand Surgery.

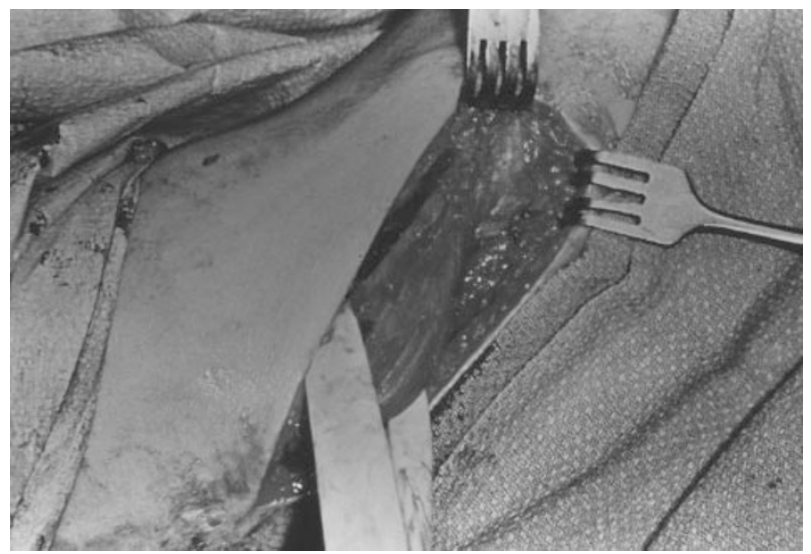

b

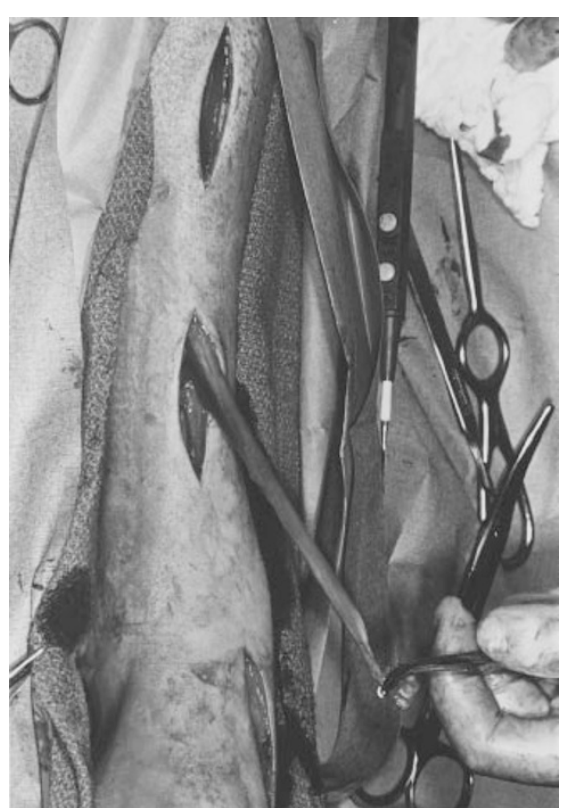

C

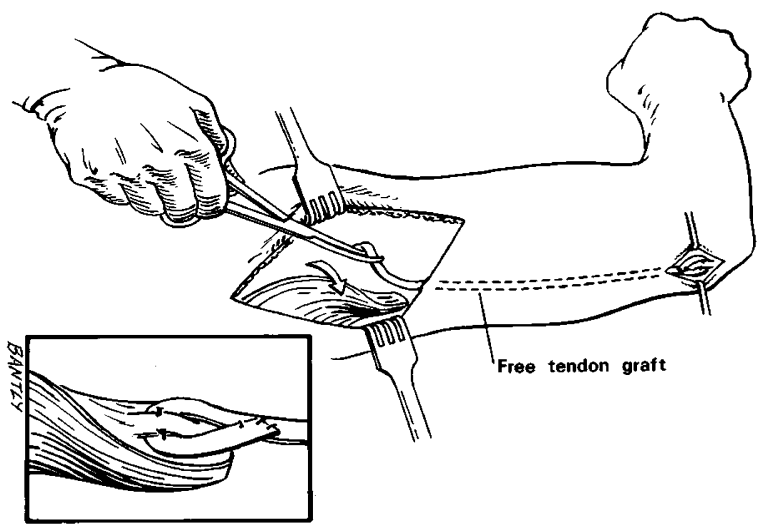

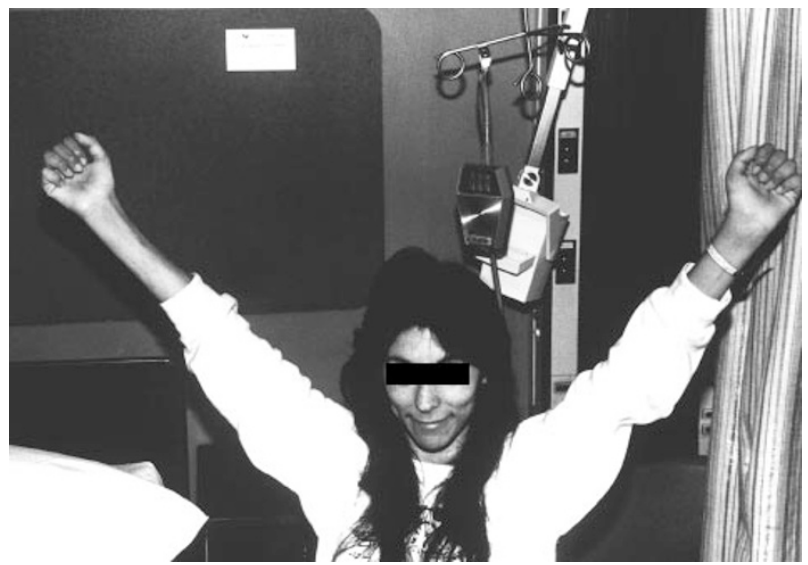

Figure 1 (a) The posterior deltoid has been mobilized and is ready to be cut with its tendinous insertion. (b) The tibialis anterior is cut at its insertion and then fed up the leg and cut when enough length is present. (c) The graft is handled with forceps and is inserted in the arm as shown. (d) She now has good extensor power in her posterior deltoid transfer 


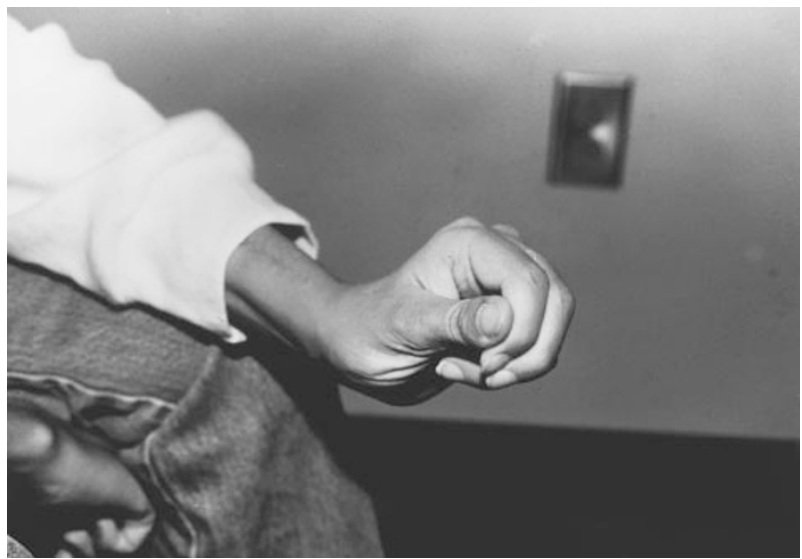

b

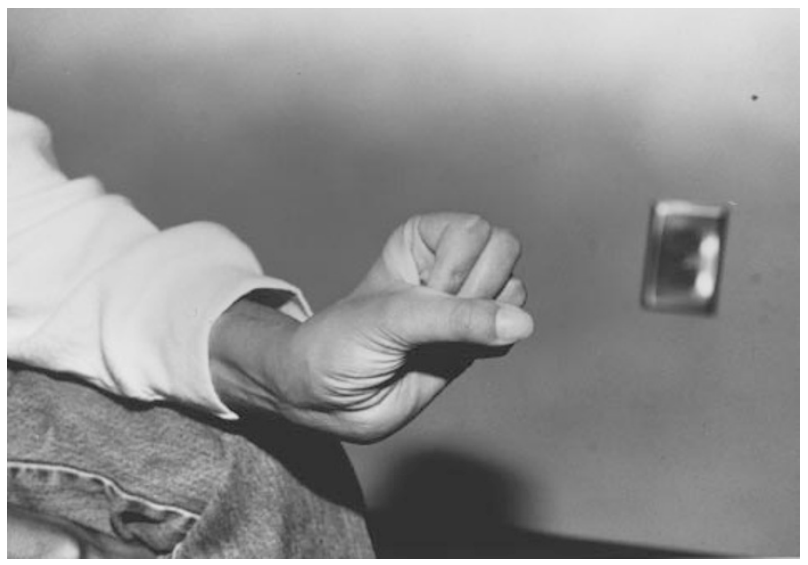

Figure 2 (a) The same patient showing opposition. (b) The same patient showing a good fist with the thumb in good opposition
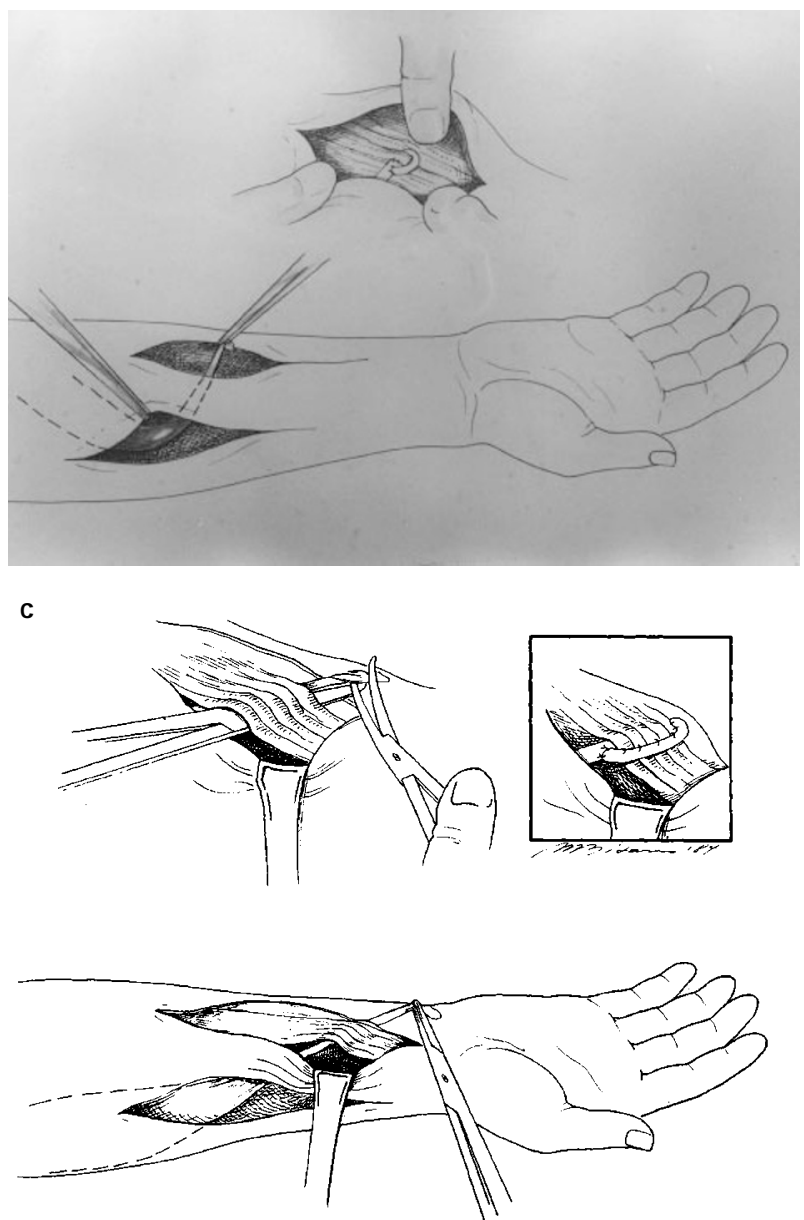

b

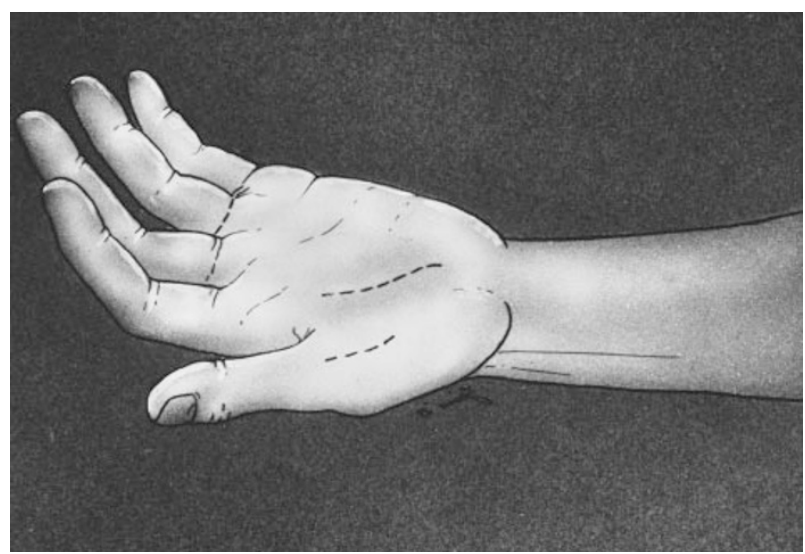

d

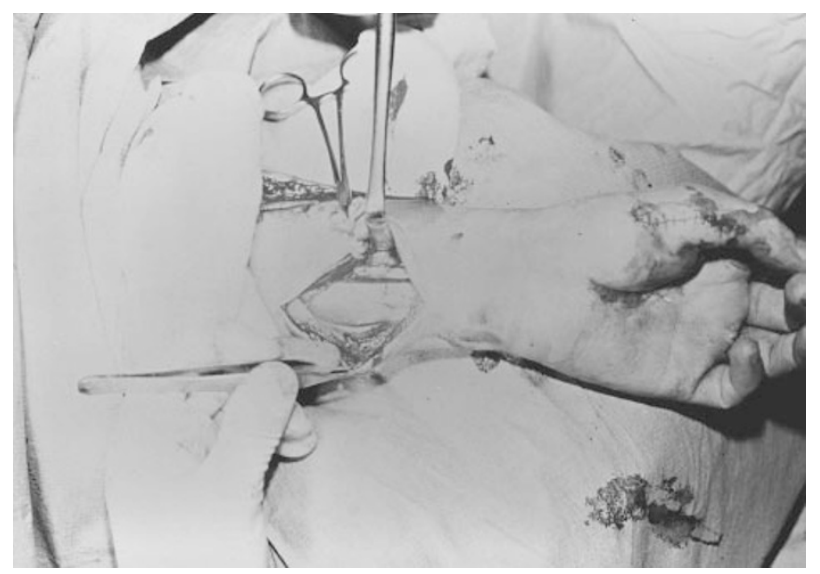

Figure 3 (a) A picture showing a diagram of a tendon being inserted into the flexor digitorum superficialis of the ring finger. (b) The hand shows three incisions. One is at the base of the ring finger, one is overlying the flexor retinaculum, and one is over the thumb to expose the abductor pollicis brevis. (c) A transferred tendon is put through the flexor digitorum profundus to give finger flexion. (d) This figure shows the unity of the flexor digitorum profundus except for the separated tendon to the index finger which was seen in most cases 
a

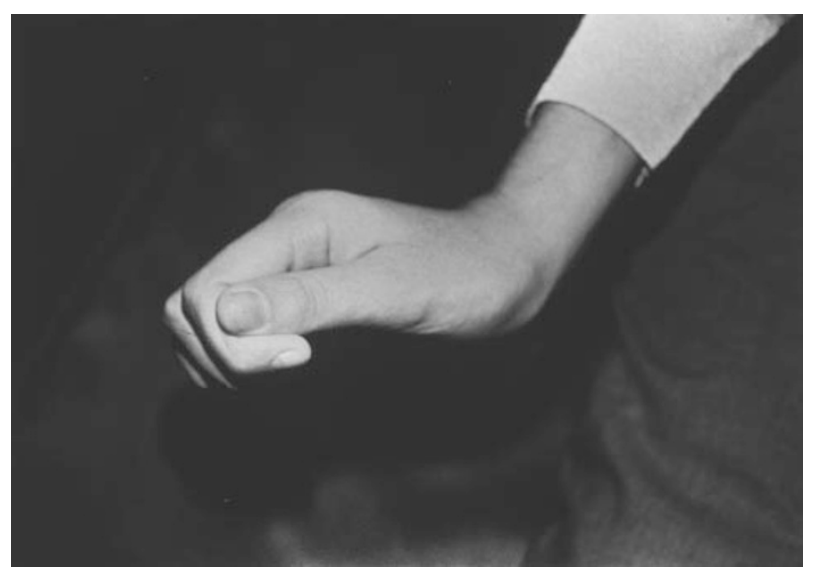

b

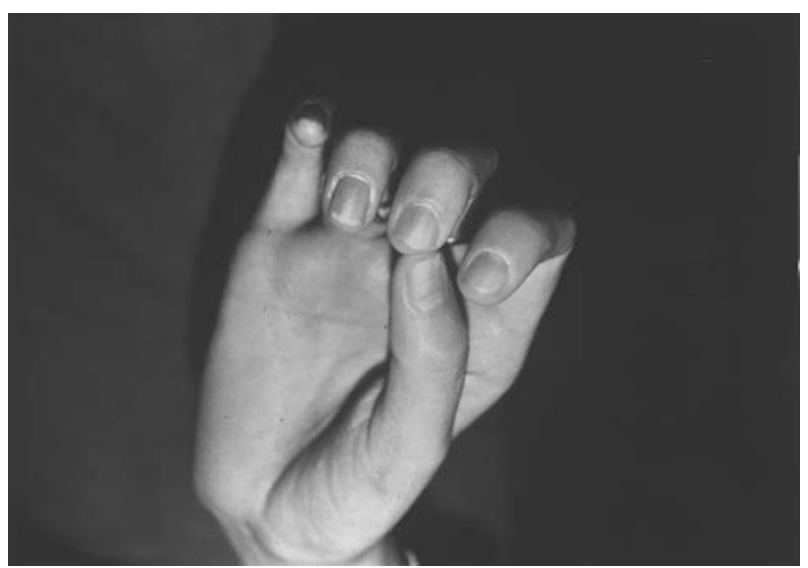

Figure 4 (a) The same patient can do key pinch with her opponens transfer. (b) The same patient can do palmar pinch

\section{Discussion}

Opponens transfer was recommended for all of the patients where it was indicated. Most activities are done with opposition and not key pinch. Most of the patients developed key pinch in addition to opposition even though opposition was the goal. All normal persons have key pinch which is twice as strong as opposition. This strength differential has convinced all surgeons to perform key pinch operations. Unfortunately one does not get opposition with the key pinch procedure.

The opponens transfer that was done was first described by Royle ${ }^{9}$ and was then modified by $\mathrm{T}$ Campbell Thompson. ${ }^{10}$ This combination of procedures has been modified by Daniel Riourdan which leads to a very flexible result. It is the easiest opponens procedure to accomplish, and in the author's opinion gives the best result. ${ }^{11}$

The posterior deltoid transfer has been simplified and made much better by the author. Immobilization is for four weeks, the elbow is immobilized at 40 degrees from complete extension, a cast from the wrist a

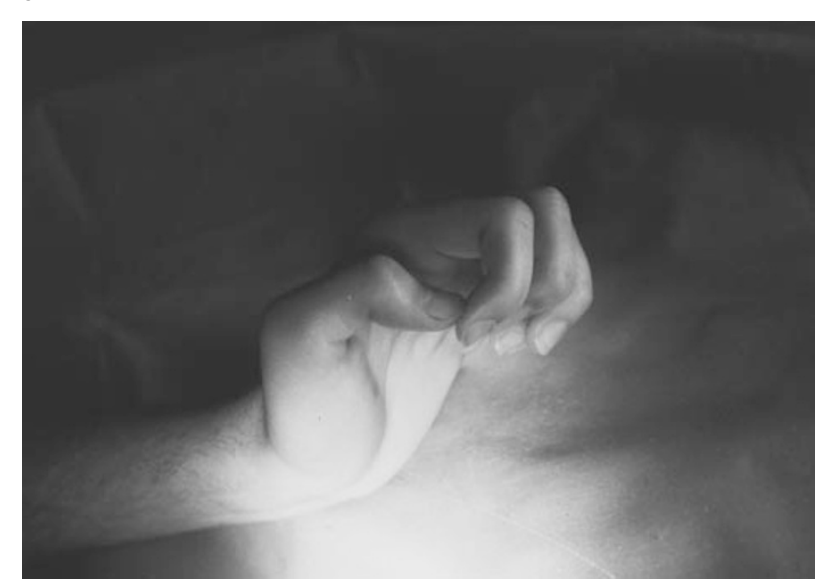

b

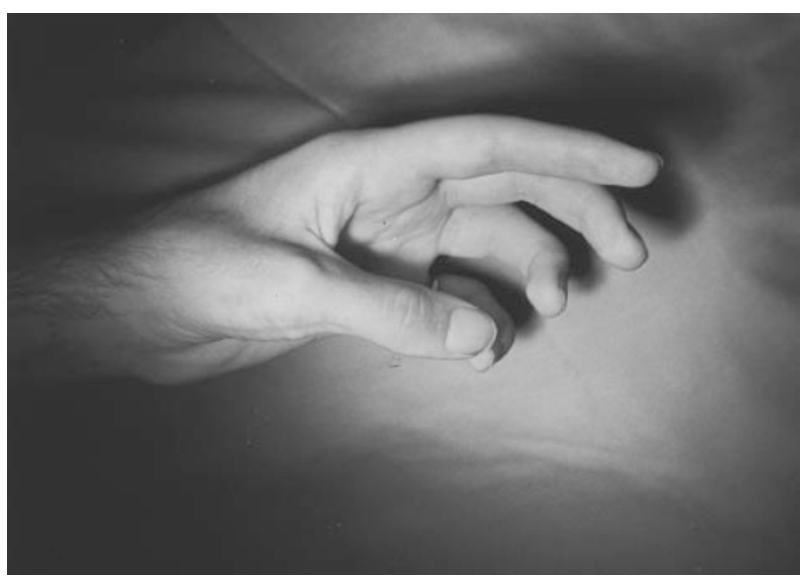

Figure 5 (a) The appearance of a hand of a patient with a neurological level of $\mathrm{C} 8$. There is paralysis of the hand intrinsics. (b) The opponens transfer allows the patient to get his thumb to his little finger

to the upper arm is applied for four weeks, the tibialis anterior or the fascia lata are used for the tendon graft, active exercises are begun immediately after removal of the cast, and a splint is applied for four weeks to protect the transfer. The splint is removed when exercises begin.

Transfers for finger flexion were very successful. One must always be careful that the index profundus is not missed. It was separated among the rest of the tendons in all cases except one patient who had two radial tendons separated. The tendons of the flexor digitorum profundus are all bound together in the forearm except the radial tendon which is clearly separated.

All incisions in the arms, forearms, thighs, legs and feet were longitudinal. This gives the best exposure and the least scarring after surgery.

All of the wrist extensors have different moment arms and therefore the transfer for wrist extension should go 
to one tendon. The author puts the extensor carpi radialis longus into the extensor carpi radialis brevis along with the brachioradialis. So the extensor carpi radialis brevis remains as the major wrist extensor.

When the pronator teres is present it should be used instead of the extensor carpi radialis longus. It probably works better than any other muscle for an opponens transfer. The brachioradialis is best for finger flexion.

The flexor carpi radialis is usually present in the group IV patients, but, using it as a transfer leads to a great deal of wrist flexor weakness usually missed by the patients. All patients following tendon transfers have a significant muscle imbalance. This is because there are not enough motors available to balance the limb. Exercises and continued use are extremely important to overcome the development of deformities. It is not important to balance the limb by doing additional surgery and eliminating functional gains following tendon transfer as long as the limb is exercised and used.

Almost all patients with C7 and C8 neurological levels can be independent for almost all activities. Patients with a C6 neurological level can be independent for almost all activities. This is based on hygiene, office, eating, and living.

\section{References}

1 Freehafer AA, von Haam E, Allen V. Tendon transfers to improve grasp after injuries to the cervical spinal cord. $J$ Bone and Joint Surg 1974; 56A: 951 - 959.

2 Freehafer AA, Kelly CM, Peckham PH. Tendon transfers for restoration of upper limb function after a cervical spinal cord injury. J Hand Surg 1984; 9A: 887-893.

3 Kelly CM, Freehafer AA, Peckham PH, Stroh K. Postoperative results of opponensplasty and flexor tendon transfer in patients with spinal cord injury. J Hand Surg 1985; 10A: 890-894.

4 Freehafer AA. Tendon transfers in patients with cervical spinal cord injury. J Hand Surg 1991; 16A: $804-809$.

5 Moberg E. Surgical treatment for absent single-hand grip and elbow extension in quadriplegia. J Bone and Joint Surg 1975; 57A: $196-206$.

6 Freehafer AA, Peckham PH, Keith MW, Mendelson LS. The brachioradialis anatomy properties and value for tendon transfer in the tetraplegic. J Hand Surg 1988; 13A: 99-104.

7 Merle d'Aubigne R, Seddon HJ, Hendy AM, Brooks DM. Symposium on reconstructive surgery of the paralysed upper limb. Proc Roy Soc Med 1949; 42: 831-844.

8 Boyes JH Bunnell's surgery of the hand 4th ed JB Lippincott: Philadelphia 1964 pp 14 and 581.

9 Royle NE. An operation for paralysis of the intrinsic muscles of the thumb. JAMA 1938; 111: $612-613$.

10 Thompson TC. Modified operation for opponens paralysis. $J$ Bone and Joint Surg 1942; 24: 632-640.

11 Riordan DC. Tendon transfers in hand surgery. J Hand Surg 1987; 8: $748-753$. 